\title{
Poster: Investigating One-Eyed and Stereo Cursors for 3D Pointing Tasks
}

\author{
Robert J. Teather * \\ Wolfgang Stuerzlinger ${ }^{\dagger}$ \\ Dept. of Computer Science \& Engineering, York University
}

\begin{abstract}
We compared two remote pointing techniques to two mouse pointing techniques using both with a stereo- and mono-rendered cursor. These were compared using a Fitts' law pointing experiment with varying target depths in a 3D scene. Results indicate that mouse-based techniques performed best and that the one-eyed cursor is beneficial only for some pointing techniques.
\end{abstract}

KeywoRDS: Pointing, 3D cursors, selection, Fitts' law.

INDEX TERMS: H.5.2 [Information Interfaces and Presentation]: User Interfaces - input devices, interaction styles

\section{INTRODUCTION}

Stereo 3D cursors introduce issues of diplopia and cue conflicts, for example, if the cursor occludes geometry extending in front of its movement plane. Ware and Lowther's "one-eyed cursor" [9] is a mono-rendered cursor which eliminates these problems altogether. They report that the one-eyed cursor outperforms a stereo 3D cursor in 3D pointing tasks. Since graphics drivers now support stereo 3D in software that was originally non-stereo, issues of cursor rendering arise. These usually display a stereorendered non-perspective cursor using the disparity of the closest occluded surface. A similar idea is to use a sliding 3D cursor that always maintains contact with the background via mouse ray casting. It handles both diplopia and stereo conflicts, as the stereo cursor simply slides across it at the same depth. We investigate if the one-eyed cursor is beneficial for such a technique, and for remote pointing techniques.

\section{Related Work}

Ray-based pointing techniques work with both 2DOF devices, and $3 / 6 \mathrm{DOF}$ devices. There is still interest in these techniques in $3 \mathrm{D}$ user interface research [3, 6, 7]. A drawback of ray-based techniques is the relative difficulty in selecting remote objects [6]. Far objects take up proportionally less screen space due to perspective, but are also proportionally closer together. According to Fitts' law [2], pointing at screen-plane projections (object images) of same-depth targets should thus be unaffected by object depth. On the other hand, 6DOF ray control has higher angular precision up close, and closer objects can be treated as effectively larger than far objects [6].

Recent work [1,5] investigated eye- and device-centric rays. Results of these studies are somewhat contradictory. One reports that device-centric rays perform better for 2D pointing tasks [5]. The other reports a new eye-centric ray technique outperforms traditional (device-centric) ray-casting [1].

We evaluate the difference between eye-centric and devicecentric rays using both one-eyed and stereo cursors using Fitts' law [2], a model of the speed/accuracy tradeoff in pointing tasks. The model is $M T=a+b \times \log _{2}(D / W+1)$. MT is movement time, $D$ is

\footnotetext{
* email: rteather@cse.yorku.ca

$\dagger$ email: wolfgang@cse.yorku.ca

IEEE Symposium on 3D User Interfaces 2012

4-5 March, Orange County, CA, USA

978-1-4673-1205-9/12/\$31.00 @2012 IEEE
}

target distance, and $W$ is target size, while $a$ and $b$ are empirically derived. An extension [4] is a post-experiment correction to adjust the error rate to $4 \%$ by re-sizing targets to their "effective" width $\left(W_{e}\right)$. The advantage of the extension is that it allows computation of throughput. Variability between throughput scores for a condition tends to be low. Consequently, results of pointing studies are more consistent and comparable [8]. Conversely, measures such as movement time vary greatly at the expense of accuracy. Details of computing throughput for 3D pointing tasks can be found in our previous work [8].

\section{Pointing Techniques}

We used two different cursor modes and compared the mouse to a remote pointing device. The first cursor mode displayed a screen plane cursor. The second mode used a sliding cursor [8]. Figure 1 depicts the four device/cursor combinations: a) a screen-plane (standard) mouse cursor, b) a sliding 3D mouse cursor, c) a novel screen-plane ray-controlled cursor, and d) a device-centric ray with sliding cursor (classic ray pointing). The "mouse" condition used the eye ray through the screen cursor for selection. The "sliding mouse cursor" displayed the cursor at the scene/ray intersection point; the cursor thus slides over geometry.

The novel remote "ray-screen" technique displays the cursor in the screen plane at the intersection of the device ray and screen. However, it uses the eye ray through this cursor for actual selection. This affords selection of object projections. This differs from Argelaguet's [1] RCE technique which uses device orientation to control eye-ray orientation. The final technique used "traditional" ray-casting: a device-centric ray that requires users to point the device directly at the $3 \mathrm{D}$ targets.

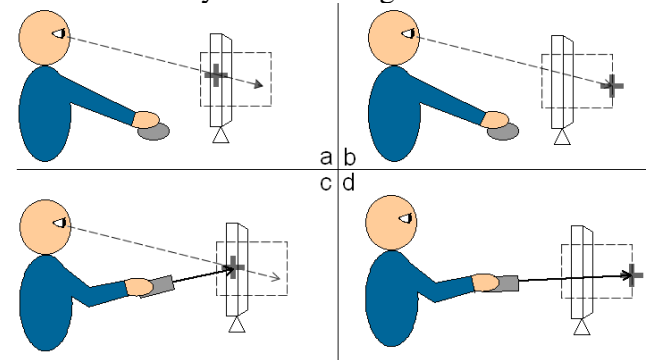

Figure 1. (a) Mouse cursor, (b) Sliding mouse cursor, (c) rayscreen, (d) ray. The dashed arrow is the selection ray, and the solid arrow is the device ray. The " + " is the cursor.

\section{Methodology}

We recruited sixteen participants, (mean age 23.1 years, eight female, all right-handed). The study used a stereo-capable PC and a NaturalPoint Optitrack calibrated to $0.7 \mathrm{~mm}$ RMS with latency of $65 \mathrm{~ms}$. The task used a 3D version of the ISO task, and requires selecting the highlighted target. The software and procedure was the same as that detailed in our previous work [8]. The study used a $4 \times 2 \times 4$ within-subjects design. The factors were technique (mouse cursor, sliding cursor, ray screen, ray), cursor (one-eyed, or stereo), and target depth $(+8,0,-8,-20 \mathrm{~cm})$. The dependent variables were time (ms), error rate (missed target percent), and throughput (bits per second). 


\subsection{Results}

Results were analyzed using repeated measures ANOVA and Tukey-Kramer multiple comparisons. All ANOVA $F$ and $p$ values are given in tables. Both mouse techniques were significantly faster than the remote techniques. The ray screen technique was significantly faster than the standard ray $(\mathrm{p}<.05)$, see Table 1 and Figure 2. Both mouse techniques had significantly lower error rates than both remote techniques. The one-eyed cursor increased error rates with the ray, see Table 2 and Figure 3.Throughput for the mouse conditions was around 4 bits per second, consistent with 2D pointing literature. This fell for targets at $-20 \mathrm{~cm}$ depth while using the 3D cursor. This fall-off is also present for the ray screen condition. Ray screen afforded significantly higher throughput than the standard ray. The one-eyed cursor hindered the ray technique, which was the worst condition overall, see Table 3, and Figure 4.

Table 1. Movement time results, significance shown with "*".

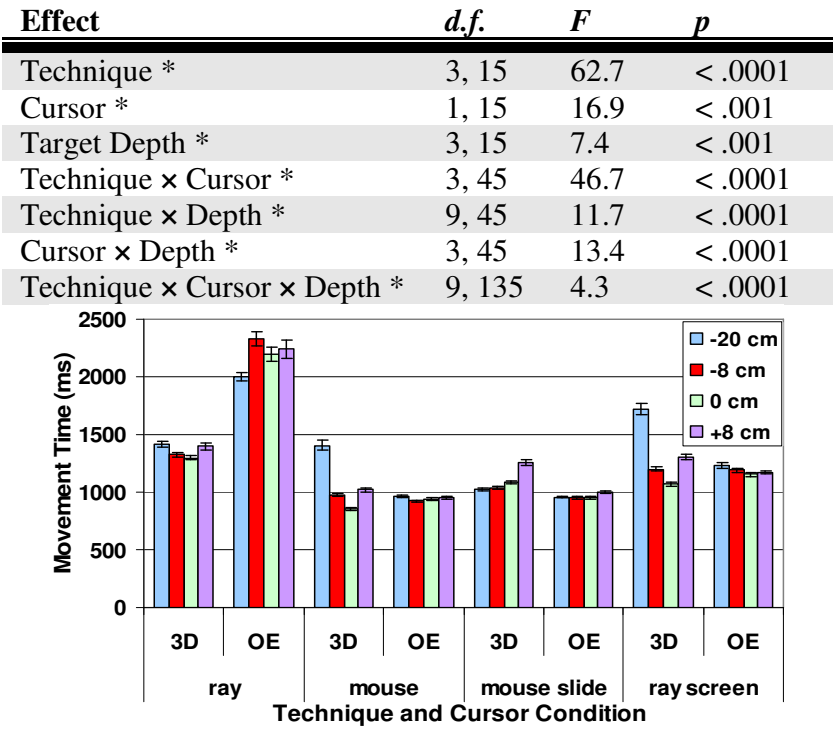

Figure 2. Movement time by condition. "OE" indicates one-eyed cursor, while "3D" indicates stereo cursor. Error bars show \pm 1 S.E.

Table 2. Error rate results, significance shown with "*”.

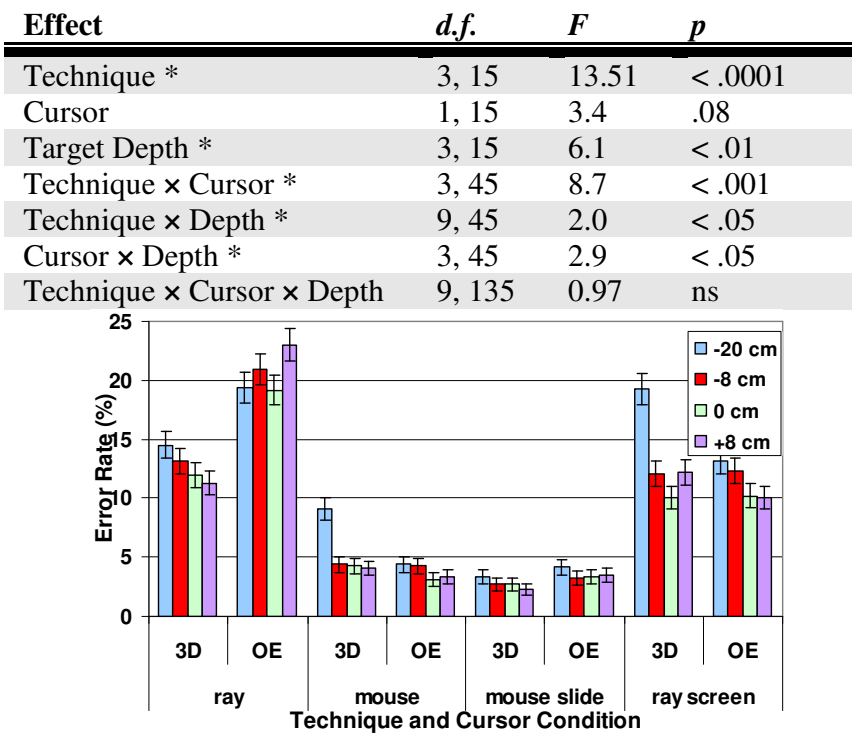

Figure 3. Error rates for each condition. Error bars show \pm 1 S.E.
Table 3. Throughput results, significance shown with “*”.

\begin{tabular}{lllc} 
Effect & d.f. & $\boldsymbol{F}$ & $\boldsymbol{p}$ \\
\hline \hline Technique * & 3,15 & 103.1 & $<.0001$ \\
Cursor & 1,15 & 0.26 & $\mathrm{~ns}$ \\
Target Depth $*$ & 3,15 & 18.1 & $<.0001$ \\
Technique $\times$ Cursor $*$ & 3,45 & 52.0 & $<.0001$ \\
Technique $\times$ Depth $*$ & 9,45 & 7.7 & $<.0001$ \\
Cursor $\times$ Depth $*$ & 3,45 & 5.3 & $<.005$ \\
Technique $\times$ Cursor $\times$ Depth * & 9,135 & 4.9 & $<.0001$
\end{tabular}

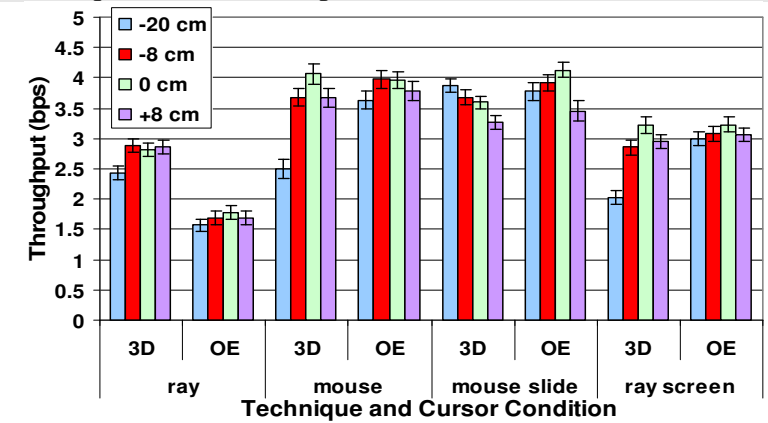

Figure 4. Throughput for each condition. Error bars show \pm 1 S.E. Higher throughput is better.

\section{Discussion/CONCLUSIONS}

Both screen plane techniques outperformed the ray, similar to Argelaguet's results [1], but contrary to Jota's [5]. We thus recommend this style of image plane technique. The multiple interaction effects indicate that only certain techniques work with a one-eyed cursor - others require a stereo cursor. Deeper targets hindered the screen-plane stereo cursor conditions but this effect was eliminated by the presence of the one-eyed cursor. The movement time scores confirm that performance is unaffected by the perspective scaling of a scene with targets displayed at the same depth when using screen-plane techniques. According to Fitts' law this makes sense, as perspective scales both the distance and width by the same amount and thus the ratio between them (the index of difficulty) remains constant.

\section{REFERENCES}

[1] Argelaguet, F. and Andujar, C., Efficient 3D pointing selection in cluttered virtual environments, IEEE Computer Graphics and Applications, 29, 2009, 34-43.

[2] Fitts, P. M., The information capacity of the human motor system in controlling the amplitude of movement, Journal of Experimental Psychology, 47, 1954, 381-391.

[3] Grossman, T. and Balakrishnan, R., The design and evaluation of selection techniques for 3D volumetric displays, UIST 2006, 3-12.

[4] ISO 9241-9 Ergonomic requirements for office work with visual display terminals (VDTs) - Part 9: Requirements for non-keyboard input devices, International Organization for Standardization, 2000.

[5] Jota, R., Nacenta, M. A., Jorge, J. A., Carpendale, S., and Greenberg, S., A comparison of ray pointing techniques for very large displays, Graphics Interface 2010, 269-276.

[6] Kopper, R., Bowman, D. A., Silva, M. G., and McMahan, R. P., A human motor behavior model for distal pointing tasks, International Journal of Human-Computer Studies, 68, 2010, 603-615.

[7] Kunert, A., Kulik, A., Lux, C., and Fröhlich, B., Facilitating system control in ray-based interaction tasks, ACM VRST 2009, 183-186.

[8] Teather, R. J. and Stuerzlinger, W., Pointing at 3D targets in a stereo head-tracked virtual environment, IEEE 3D UI 2011, 87-94.

[9] Ware, C. and Lowther, K., Selection using a one-eyed cursor in a fish tank VR environment, ACM TOCHI, 4, 1997, 309-322. 